\title{
Modulational Instability for Nonlinear Schrödinger Equations With a Periodic Potential.
}

\author{
Jared C. Bronski and Zoi Rapti \\ Communicated by Charles Li, received June 20, 2005.
}

\begin{abstract}
We study the linearized stability properties of periodic solutions to the nonlinear Schrödinger (NLS) equation with a periodic potential. We exploit the symmetries of the problem, in particular the Hamiltonian structure and the $\mathbb{U}(1)$ symmetry, to develop a simple sufficient condition that guarantees the existence of a modulational instability spectrum along the imaginary axis. In the case of small amplitude solutions that bifurcate from the band edges of the linear problem this condition becomes especially simple. We find that the small amplitude solutions corresponding to the band edges alternate stability, with the first band edge being modulationally unstable in the focusing case, the second band edge being modulationally unstable in the defocusing case, and so on. This small amplitude result has a nice physical interpretation in terms of the effective mass of a particle in the periodic potential. We also consider, in somewhat less detail, some sideband instabilities in the small amplitude limit. We find that, depending on the Krein signature of the collision, these instabilities can be of one of two types. Finally we illustrate these results in the case where the potential $V(x)$ is an elliptic function, where many of the relevant calculations can be done explicitly.
\end{abstract}

\section{Contents}

1. Introduction 336

2. Fundamentals of Hamiltonian Floquet Theory 337

3. Modulational Instability of Standing Waves 341

4. Finite Wavelength Instabilities. 347

5. Explicit Examples and Numerics 349

6. Conclusions 353

References $\quad 354$

1991 Mathematics Subject Classification. Primary 35Q55,37K45; Secondary 35B27, 35P15.

Key words and phrases. Linear Stability, Krein Signature, non-selfadjoint eigenvalue problems.

The first author was supported in part by NSF Grants DMS 0203938 and DMS0354373.

(c)2005 International Press 


\section{Introduction}

In this paper we consider the stability of standing wave solutions to the NLS equation with an external potential:

$$
i \psi_{t}=-\frac{1}{2} \psi_{x x} \pm \epsilon|\psi|^{2} \psi+V(x) \psi,
$$

where the potential $V(x)$ has period 1. Such a model arises in a number of physical contexts, most notably as a simplified model for Bose-Einstein condensates in a periodic traps. In this context the presence or absence of a dynamical instability is believed to have important physical consequences $[\mathbf{2 6}]$.

We assume that we have a standing wave solution $\psi(x, t, \epsilon)=\exp (-i \omega(\epsilon) t) \phi(x, \epsilon)$, where $\phi(x, \epsilon)$ is real valued and either periodic, $\phi(x+1, \epsilon)=\phi(x, \epsilon)$, or antiperiodic, $\phi(x+1, \epsilon)=-\phi(x, \epsilon)$. We can assume that the standing wave has fixed $\mathbf{L}_{2}$ norm, since this amounts to rescaling $\epsilon$. For certain perturbative results we will also assume that the standing wave bifurcates continuously from the FloquetBloch eigenfunctions in the usual way. In other words we assume that $\phi(x, 0)$ is an eigenfunction of the periodic Schrödinger operator

$$
\mu \phi=\omega(0) \phi=-\frac{1}{2} \phi_{x x}+V(x) \phi
$$

corresponding to a band edge, and $\phi(x, \epsilon) \in C^{2}([0,1] \times(-\delta, \delta))$. Such a result follows from standard variational arguments: see, for instance, the work of Crandall and Rabinowitz[5] and Rabinowitz[21].

This problem differs from the solitary wave stability problem because the spectrum of the linearized operator consists of bands of absolutely continuous spectrum. As a result of this index results based on counting the dimensions of the negative subspaces of $\mathbf{L}_{ \pm}$, like those of Jones[10], Grillakis[8], or Grillakis, Shatah and Strauss[9] are no longer applicable in a straightforward way. We shall see that in many cases the above standing wave can be shown to be linearly unstable for arbitrarily small amplitudes. This stands in marked contrast to the problem on the whole line, where Rose and Weinstein[22] have shown that solutions which bifurcate from the linear solutions are stable for sufficiently small amplitudes.

We find that the combination of the Hamiltonian structure and the $\mathbb{U}(1)$ symmetry dramatically simplifies the structure and possible bifurcations of the spectrum of the linearized operator. We find a simple sufficient condition on the $\mathbf{L}_{+}$ operator which guarantees the existence of a modulational instability. In the case of weakly nonlinear standing waves, when $\epsilon$ is small, we apply a perturbation argument to show that the lower band edges are modulationally unstable in the focusing case, while the upper band edges are modulationally unstable in the defocusing case.

We also study, in somewhat less detail, some bifurcations which occur for nonzero values of $\mu$ when a certain eigenvalue degeneracy condition is met. We find that this bifurcation generically leads to the emergence of complex eigenvalues, and thus instability. Depending on the Krein signature of the unperturbed eigenvalues this may or may not lead to the opening of a gap along the real axis.

There have been a number of papers in the literature which have used the Hamiltonian structure of the linearized operator to garner information on the structure of the linearized spectrum and the possible instabilities of standing waves. We will try to mention a few which are most closely related to the calculation presented here. One of the earliest such papers that we are aware of is the work of 
MacKay and Saffman $[\mathbf{1 4}, \mathbf{1 5}]$ on the stability of water waves. MacKay and Saffman made extensive use of the Hamiltonian structure, primarily the Krein sign, in order to classify the possible bifurcations of the linearized spectrum for water waves. More recently Carter and Segur[4] carried out a related study on the stability of cnoidal wave solutions to the two dimensional cubic NLS. Pelinovsky, Sukhurukhov, and Kivshar[20] have studied the bifurcations and stability of gap solitons for an NLS type equation with a periodic potential. Sandstede and Yew[23] have studied the same problem we consider here, the stability of periodic solutions to a nonlinear Schrodinger equation with a potential, in the limit of long period (the tight-binding approximation). Finally, Li and McLaughlin[13] and Mityagin[17] have made a study of the periodic non-selfadjoint Zakharov-Shabat operator, and Shin has made a study of the spectrum of a non-self-adjoint periodic second order Schrodinger equations[24].

Throughout this paper we will apply the following notation. $\mathbf{U}(x)$ will denote the solution operator of the periodic ODE. The matrix $\mathbf{M}$ will denote the monodromy, or period, map $\mathbf{U}(1)$. We will have occasion to consider the spectral properties of the second order operators $\mathbf{L}_{ \pm}$individually. The matrix $\mathbf{m}$ will denote the mondromy matrix of the second order eigenvalue problem associated to $\mathbf{L}_{ \pm}$. For each of these quantities the dependence on the parameters $\mu, \epsilon$ will be generally be suppressed unless it is necessary for clarity. Similarly $K_{ \pm}(\mu)$ will denote the Floquet discriminants of the full stability problem, while $k(\mu)$ will denote the Floquet discriminant of the second order problem associated with $\mathbf{L}_{ \pm}$.

\section{Fundamentals of Hamiltonian Floquet Theory}

In this section we review some results of Floquet theory for the special case in which the equations admit a Hamiltonian formulation. For more details see the text of Yakubovich and Starzhinskii[27].

2.1. The Lyapunov-Poincare theorem. To begin we assume a Floquet problem of the following form:

$$
\begin{aligned}
\mathbf{U}_{x}= & \mathbf{J} \mathbf{H}(x) \mathbf{U} \mathbf{U}(0)=\mathbf{I}_{2 N \times 2 N} \\
& \mathbf{H}(x+1)=\mathbf{H}(x) \mathbf{H}^{t}=\mathbf{H}
\end{aligned}
$$

where, for simplicity, we have set the period to one. Here $\mathbf{I}_{2 N \times 2 N}$ is the $2 N \times 2 N$ identity matrix and $\mathbf{J}$ is the usual skew-symmetric matrix

$$
\mathbf{J}=\left(\begin{array}{cc}
0 & -\mathbf{I}_{N} \\
\mathbf{I}_{N} & 0
\end{array}\right) .
$$

The monodromy matrix $\mathbf{M}$ is defined to be the period map $\mathbf{M}=\mathbf{U}(1)$. It is easy to see that $\mathbf{U}(x)$, and thus $\mathbf{M}$, satisfies the relation

$$
\mathbf{U}^{t} \mathbf{J} \mathbf{U}=\mathbf{J},
$$

so that $\mathbf{U}(x)$ is a symplectic ${ }^{1}$ matrix. From this it follows that $\operatorname{det}(\mathbf{U})=1$, and more generally that the characteristic polynomial $P(\lambda)=\operatorname{det}(\mathbf{U}-\lambda \mathbf{I})$ satisfies

$$
\begin{aligned}
\operatorname{det}(\mathbf{U}-\lambda \mathbf{I}) & =\operatorname{det}\left(\mathbf{U}^{t}-\lambda \mathbf{I}\right)=\operatorname{det}\left(\mathbf{J}\left(\mathbf{U}^{t}-\lambda \mathbf{I}\right) \mathbf{J}^{t}\right) \\
& =\operatorname{det}\left(\mathbf{U}^{-1}-\lambda \mathbf{I}\right)=\lambda^{2 N} \operatorname{det}\left(\mathbf{U}^{-1}\right) \operatorname{det}\left(\mathbf{U}-\lambda^{-1} \mathbf{I}\right) \\
& =\lambda^{2 N} P\left(\lambda^{-1}\right)
\end{aligned}
$$

\footnotetext{
${ }^{1}$ Sometimes called J-orthogonal.
} 
thus the polynomial is palindromic - if $P(\lambda)=\sum_{0}^{2 N} a_{j} \lambda^{j}$ then $a_{2 N-j}=a_{j}$. This result is known as the Lyapunov-Poincare theorem [27].

This symmetry implies that the problem of finding the roots of the $2 N^{\text {th }}$ degree polynomial $P(\lambda)$ can be reduced to finding the roots of an $N^{\text {th }}$ degree polynomial $\tilde{P}(z)$ by means of the transformation $z=\lambda+\lambda^{-1}$. For the case of the stability problem for standing wave solutions to the nonlinear Schrodinger equation the monodromy matrix is $4 \times 4(N=2)$ In the case the characteristic polynomial of the monodromy matrix takes the form

$$
P(\lambda)=1+a \lambda+b \lambda^{2}+a \lambda^{3}+\lambda^{4}=0 .
$$

Under the conformal map $z=\lambda+\lambda^{-1}$ the roots of this polynomial are mapped to the roots of the second degree polynomial

$$
\tilde{P}(z)=z^{2}+a z+(b-2)=0
$$

with $\lambda=\frac{z \pm \sqrt{z^{2}-4}}{2}$, and the characteristic polynomial admits an explicit factorization into two second degree polynomials,

$$
P(\lambda)=\left(1-K_{+} \lambda+\lambda^{2}\right)\left(1-K_{-} \lambda+\lambda^{2}\right)
$$

where the $K_{ \pm}$are the following algebraic functions of $a, b$ :

$$
K_{ \pm}=\frac{-a \pm \sqrt{a^{2}-4 b+8}}{2} .
$$

The functions $K_{+}$will be refered to as the Floquet discriminants. Since the conformal mapping $z=\lambda+\frac{1}{\lambda}$ takes the unit circle to the real interval $[-2,2]$ it follows that the monodromy matrix has two eigenvalues (counted by algebraic multiplicity) on the unit circle if $K_{+}$lies in the real interval $[-2,2]$, and two more if $K_{-} \in[-2,2]$.

It is convenient to express the $K_{+}$in terms of the invariants of $\mathbf{M}$. Since $a=-\operatorname{Tr}(\mathbf{M}), b=-\frac{1}{2} \operatorname{Tr}\left(\mathbf{M}^{2}\right)+\frac{1}{2} \operatorname{Tr}(\mathbf{M})^{2}$ the Floquet discriminants are given by

$$
K_{ \pm}=\frac{\operatorname{Tr}(\mathbf{M}) \pm \sqrt{-(\operatorname{Tr}(\mathbf{M}))^{2}+2 \operatorname{Tr}\left(\mathbf{M}^{2}\right)+8}}{2}
$$

This leads to our first observation

LEMma 2.1. The possible (algebraic) multiplicities of the eigenvalues of a $4 \times 4$ monodromy matrix are $(1,1,1,1),(1,1,2),(2,2)$ and 4 . The conditions on the monodromy matrix $\mathbf{M}$ which produce eigenvalues of higher multiplicity are as follows:

\begin{tabular}{||l|l|l||}
\hline \hline Condition on $K_{ \pm}$ & Condition on $\mathbf{M}$ & Root Multiplicities \\
\hline$K_{+}= \pm 2$ or $K_{-}= \pm 2$ & $\left.\operatorname{Tr}\left(\mathbf{M}^{2}\right)=(\operatorname{Tr}(\mathbf{M}) \pm 2)\right)^{2}$ & (1,1,2) \\
Simple Band Edge & & \\
\hline$K_{+}= \pm 2$ and $K_{-}=\mp 2$ & $\operatorname{Tr}(\mathbf{M})=0$ & (2,2) \\
Repeated Band Edge & $\operatorname{Tr}\left(\mathbf{M}^{2}\right)=4$ & \\
\hline$K_{+}=K_{-} \neq \pm 2$ & $2 \operatorname{Tr}\left(\mathbf{M}^{2}\right)=(\operatorname{Tr}(\mathbf{M}))^{2}-8$ & (2,2) \\
'Accidental' Degeneracy & $\operatorname{Tr}(\mathbf{M}) \neq \pm 4$ & \\
\hline$K_{+}=K_{-}= \pm 2$ & $\operatorname{Tr}(\mathbf{M})= \pm 4$ & 4 \\
Double Band Edge & $\operatorname{Tr}\left(\mathbf{M}^{2}\right)=4$ & \\
\hline \hline
\end{tabular}

PROOF. A straightforward calculation from the explicit factorization given in (2.10). 
It is worthwhile considering the codimensions of the above possibilities. Since we have a one parameter family of mondromy matrices, parameterized by the spectral parameter $\mu$, (here we are considering $\epsilon$ as fixed) we should generically expect to see possibilities 1 and 3, which require only one condition, but not possibilities 2 and 4, which require two independent conditions be satisfied. We shall see that possibility 4 always happens at $\mu=0$ for $\epsilon=0$ due to symmetries. It is this degeneracy which forces a modulational instability in the small amplitude case. It is this situation that we will consider in most detail. We will also consider possibility 3 , in somewhat less detail. We will not consider possibility 2 , since it is non-generic, nor possibility 1 , since it is not very interesting: one expects that the band edges will typically move under perturbation.

2.2. Krein Signature. There is an extensive theory of the structural stability of symplectic matrices due to Krein and collaborators. We shall give only a brief summary of this theory here. It is clear that if a symplectic matrix $\mathbf{M}$ has $k$ distinct eigenvalues on the unit circle then it follows from continuity that a nearby symplectic matrix $\tilde{\mathbf{M}}$ must also have $k$ distinct eigenvalues on the unit circle. Thus eigenvalues of symplectic matrices can only leave the unit circle via collisions. What is less clear is that only certain collisions can lead to pairs of eigenvalues leaving the unit circle. The quantity which distinguishes such "dangerous" collisions is that of the Krein signature. The Krein sign $\eta$ of an eigenvector $\vec{v}$ of a symplectic matrix $\mathbf{M}$ is defined to be

$$
\eta=\operatorname{sgn}(<\vec{v} \mathbf{J} \vec{v}>)
$$

while the signature $(p, q)$ of an $r$-dimensional eigenspace is defined as follows: $p$ (resp. q) is the number of linearly independent eigenvectors with positive (resp. negative) Krein sign.

The fundamental stability result for symplectic matrices says that a symplectic matrix is structurally stable (i.e. the number of eigenvalues on the unit circle does not change under perturbation ${ }^{2}$ ) if all eigenspaces corresponding to eigenvalues on the unit circle have a definite Krein signature. Further this result is tight: if an eigenspace is of indefinite Krein signature then a generic perturbation will cause roots to move off of the unit circle. The first result is known as Krein's theorem, while the second is known as the Krein-Gelfand-Lidskii strong stability theorem. For precise statements, as well as proof, see Yakubovich and Starzhinskii[27].

2.3. Hill's Equation. In this section we state some properties of the Hill's equation

$$
\mathbf{H} \psi=-\psi_{x x}+Q(x) \psi=\mu \psi
$$

that will be useful in the sequel. We will also define a quantity that will be useful in the analysis to follow.

In the remainder of this section $\mathbf{m}$ is the $(2 \times 2)$ monodromy matrix associated to the Hill's equation (2.14):

$$
\mathbf{m}=\left(\begin{array}{cc}
\psi_{1}(1, \mu) & \psi_{2}(1, \mu) \\
\psi_{1}^{\prime}(1, \mu) & \psi_{2}^{\prime}(1, \mu)
\end{array}\right)
$$

\footnotetext{
${ }^{2}$ Here perturbations are always assumed to preserve the symplectic nature of the matrix.
} 
with $k(\mu)=\operatorname{Tr}(\mathbf{m})$, and $\mathbf{j}$ is the standard skew-symmetric form

$$
\mathbf{j}=\left(\begin{array}{cc}
0 & -1 \\
1 & 0
\end{array}\right) \text {. }
$$

We begin with some well-known facts. The spectrum of the above eigenvalue problem consists of the union of the set of intervals $\operatorname{spec}(\mathbf{H})=\left[\mu_{0}, \mu_{1}{ }^{\prime}\right] \cup\left[\mu_{2}{ }^{\prime}, \mu_{1}\right] \cup$ $\left[\mu_{2}, \mu_{3}^{\prime}\right] \ldots$, where $k\left(\mu_{i}\right)=2$ and $k\left(\mu_{i}^{\prime}\right)=-2$. The points $\left\{\mu_{i}\right\},\left\{\mu_{i}^{\prime}\right\}$ are referred to as the periodic and antiperiodic eigenvalues respectively, and together they comprise the band edges. It is obviously true from continuity that $\mu_{2 i}<\mu_{2 i+1}^{\prime}$ and $\mu_{2 i}^{\prime}<$ $\mu_{2 i-1}$, so the bands are always nontrivial. It can happen that $\mu_{2 i-1}^{\prime}=\mu_{2 i}^{\prime}$, or $\mu_{2 i-1}=\mu_{2 i}$, corresponding to a closed gap. This is commonly referred to as a double point. In the interior of each band there are two quasi-periodic solutions, while at the band edge there is one periodic or antiperiodic solution and one solution which grows linearly, unless the band edge is a double point, in which case there are two periodic or antiperiodic solutions. The derivative of the Floquet discriminant is non-zero in the bands, has exactly one zero in each gap, and is nonzero on the band edges unless the band edge is a double point, in which case the derivative of Floquet discriminant has a simple zero. Proofs of these facts can be found in Magnus and Winkler[16].

In the first lemma we introduce a quantity which will play an important role in the perturbation analysis. The sign of this quantity will be important in determining the modulational stability of the standing wave.

Lemma 2.2. Define the quantity $\sigma(\mu)$ as follows:

$$
\sigma(\mu)=\operatorname{Tr}(\mathbf{j m}(\mu))
$$

Then the following hold

(1) $\sigma(\mu)$ is non-zero on the interior of each band.

(2) $\sigma(\mu)$ vanishes at a band edge iff the band edge is a double point.

(3) $\operatorname{sgn}(\sigma(\mu))=-\operatorname{sgn}\left(k^{\prime}(\mu)\right)=-\operatorname{sgn}\left(\operatorname{Tr}\left(\frac{\partial \mathbf{m}}{\partial \mu}\right)\right)$ if $\mu \in \operatorname{spec}(\mathbf{H})$.

Proof. On the interior of a band it holds $k^{2}(\mu)-4<0$. If we set $\eta_{1}=\psi_{1}(1, \mu)$, $\eta_{2}=\psi_{2}(1, \mu), \eta_{1}^{\prime}=\psi_{1}^{\prime}(1, \mu)$, and $\eta_{2}^{\prime}=\psi_{2}^{\prime}(1, \mu)$, then

$$
k^{2}(\mu)-4=\left(\eta_{1}-\eta_{2}^{\prime}\right)^{2}+4 \eta_{1}^{\prime} \eta_{2},
$$

which implies that $\eta_{1}^{\prime} \eta_{2} \leq 0$. By its definition, $\sigma(\mu)=-\eta_{1}^{\prime}+\eta_{2}=-\operatorname{sgn}\left(\eta_{1}^{\prime}\right)\left(\left|\eta_{1}^{\prime}\right|+\right.$ $\left.\left|\eta_{2}\right|\right)$. But (2.18) implies that $\eta_{1}^{\prime} \neq 0$ and $\eta_{2} \neq 0$. Thus it follows that $\sigma(\mu)$ is nonzero in the interior of each band.

Let a band edge be a double point. It follows that $k^{2}(\mu)-4=0$ and moreover $k^{\prime}(\mu)=0$. Following $[\mathbf{1 6}]$ one can show that

$$
k^{\prime}(\mu)=\operatorname{sgn}\left(\eta_{1}^{\prime}\right)\left(\left|\eta_{1}^{\prime}\right| \int \psi_{2}^{2}+\left|\eta_{2}\right| \int \psi_{1}^{2} \pm 2 \sqrt{\left|\eta_{1}^{\prime}\right|} \sqrt{\left|\eta_{2}\right|} \int \psi_{1} \psi_{2}\right),
$$

where $\psi_{1}, \psi_{2}$ are the two linearly independent solutions of

$$
\psi_{x x}+(\mu-Q(x)) \psi=0
$$

that satisfy the initial conditions $\psi_{1}(0, \mu)=1, \psi_{1}^{\prime}(0, \mu)=0, \psi_{2}(0, \mu)=0$, and $\psi_{2}^{\prime}(0, \mu)=1$. We used again (2.18) and, in particular, the inequality $\eta_{1}^{\prime} \eta_{2} \leq 0$. By Cauchy-Schwartz it follows that $\operatorname{sgn}\left(\eta_{1}^{\prime}\right)=0$, thus $\eta_{1}^{\prime}=0$. But then, also $\eta_{1}-\eta_{2}^{\prime}=0$, which in turn forces $\eta_{2}=0$. 
The other implication is proved as follows: $\sigma(\mu)=0$ implies $0=k^{2}(\mu)-4=$ $\left(\eta_{1}-\eta_{2}^{\prime}\right)^{2}+4 \eta_{2}^{2}$, so $\eta_{2}=\eta_{1}^{\prime}=0$ and $\eta_{1}-\eta_{2}^{\prime}=0$. But then clearly $k^{\prime}(\mu)=0$.

Finally, again in [16] it is shown that if $\eta_{1}^{\prime} \neq 0$ then $k^{\prime}(\mu)$ and $\eta_{1}^{\prime}$ have the same sign. The result follows, since from the first part of this lemma, $\sigma(\mu)$ and $\eta_{1}^{\prime}$ have opposite signs.

This quantity is related to the Krein sign, and actually agrees with the Krein sign of the eigenvalue of the monodromy matrix in the upper half plane. However the main utility of this quantity is that it allows one to compute the sign of the off-diagonal piece of the Jordan normal form at a band edge, as in the lemma below.

Lemma 2.3. At a band edge the monodromy matrix $\mathbf{m}$ has the following Jordan normal form:

$$
\mathbf{m}=\mathbf{o}^{t}\left(\begin{array}{cc} 
\pm 1 & \sigma(\mu) \\
0 & \pm 1
\end{array}\right) \mathbf{o}
$$

where $\mathbf{o}$ is a proper orthogonal matrix: $\mathbf{o o}^{\mathbf{t}}=\mathbf{I}, \operatorname{det}(\mathbf{o})=+1$

Proof. It is known that at a band edge that is not a double point \pm 1 is an eigenvalue of algebraic multiplicity two, and geometric multiplicity 1 . The Jordan normal form implies that

$$
\mathbf{m}=\mathbf{o}^{t}\left(\begin{array}{cc} 
\pm 1 & K \\
0 & \pm 1
\end{array}\right) \mathbf{o}=\mathbf{o}^{t} \tilde{\mathbf{m}} \mathbf{o}
$$

where $\mathbf{o}$ is orthogonal and can be chosen to have determinant +1 . It remains to be checked that $K=\sigma(\mu)$. One easily observes that $\mathbf{j}$ is a rotation and thus commutes with o. This implies that $\sigma=\operatorname{Tr}(\mathbf{j m})=\operatorname{Tr}\left(\mathbf{o j o}^{\mathbf{t}} \tilde{\mathbf{m}}\right)=K$, so $\sigma$ is constant on the connected component of the orthogonal group containing the identity.

REMARK 2.4. If $\mathbf{o}$ is chosen to be on the other connected component of the orthogonal group $\mathbf{j}$ and $\mathbf{o}$ anti-commute, and the sign of the $\sigma$ is reversed. This is not surprising, since $\sigma$ represents a sense of rotation of the monodromy matrix, and thus satisfies a right-hand rule. The value of $\sigma$ at a band edge, in particular the sign, will prove important in determining the stability of small amplitude standing wave solutions. Note that the Krein signature of an eigenvalue of the monodromy matrix in the upper half-plane is equal to the sign of $\sigma$, and thus has the opposite sign from the derivative of the Floquet discriminant. Thus the sign of this quantity is positive in the first band, negative in the second band, and generally $\operatorname{sign}(\sigma)=(-1)^{k-1}$, where $k$ is the band number.

\section{Modulational Instability of Standing Waves}

3.1. General Results. We assume that (for $\epsilon$ fixed) we have a solution $\psi=$ $e^{-i \omega(\epsilon) t} \phi_{\text {stand }}(x, \epsilon)$ to the NLS equation with a periodic potential

$$
\begin{aligned}
i \psi_{t} & =-\frac{1}{2} \psi_{x x}+\epsilon|\psi|^{2} \psi+V(x) \psi \\
V(x+1) & =V(x),
\end{aligned}
$$


where $\phi$ is real and either periodic $\phi(x+1)=\phi(x)$ or anti-periodic $\phi(x+1)=-\phi(x)$. The eigenvalue problem governing the linearized stability is given by

$$
\begin{aligned}
& \mathbf{L}_{+} p=\mu q \\
& \mathbf{L}_{-} q=\mu p
\end{aligned}
$$

where the operators $\mathbf{L}_{ \pm}$are given by

$$
\begin{aligned}
& \mathbf{L}_{-}=-\frac{1}{2} \partial_{x x}+V(x)+\epsilon\left|\phi_{\text {stand }}\right|^{2}(x, \epsilon)-\omega(\epsilon) \\
& \mathbf{L}_{+}=-\frac{1}{2} \partial_{x x}+V(x)+3 \epsilon\left|\phi_{\text {stand }}\right|^{2}(x, \epsilon)-\omega(\epsilon)
\end{aligned}
$$

Here we have eliminated the time dependence via a Fourier transform, so that an instability corresponds to an eigenvalue $\mu$ off of the real axis. We note in passing that all of these results carry over in a straightforward way to a more general nonlinear Schrodinger equation of the form

$$
i \psi_{t}=-\frac{1}{2} \psi_{x x}+V(x) \psi+U\left(x,|\psi|^{2}\right) \psi
$$

which has $\mathbf{L}_{ \pm}$operators given by

$$
\begin{aligned}
& \mathbf{L}_{-}=-\frac{1}{2} \partial_{x x}+V(x)+U\left(x,\left|\phi_{\text {stand }}\right|^{2}\right)-\omega \\
& \mathbf{L}_{+}=-\frac{1}{2} \partial_{x x}+V(x)+U\left(x,\left|\phi_{\text {stand }}\right|^{2}\right)+2 D_{2} U\left(x,\left|\phi_{\text {stand }}\right|^{2}\right)-\omega .
\end{aligned}
$$

For ease of discussion we consider only the cubic case, which is physically the most interesting.

The eigenvalue problem in Eqn (3.6) has a Hamiltonian formulation for real $\mu$. The Lyapunov-Poincare theorem of the previous section implies that the characteristic polynomial of the monodromy matrix is palindromic, $P(\lambda)=\lambda^{4} P\left(\lambda^{-1}\right)$, for $\mu$ on the real axis. Also note that for arbitrary values of $\epsilon$ the spectrum is symmetric about the real and imaginary axes, since the eigenvalue problem is invariant under the transformations $\mu \rightarrow-\mu, p \rightarrow-p, q \rightarrow q$ and $\mu \rightarrow \bar{\mu}, p \rightarrow \bar{p}, q \rightarrow \bar{q}$.

We begin by noting a few simple properties of this eigenvalue problem, and the associated monodromy matrix. The notation follows that of section 2.1.

Proposition 3.1. The Floquet stability problem for an NLS standing wave has the following properties:

(1) $\mu=0$ is a periodic (anti-periodic) eigenvalue.

(2) $\mathbf{M}(\mu)$ is an entire matrix-valued function of $\mu$, of order $\frac{1}{2}$.

(3) The monodromy matrix $\mathbf{M}$ is symplectic for all $\mu \in \mathbf{C}$.

(4) The Floquet discriminants $K_{ \pm}(\mu)$ are analytic functions of $\mu$ away from the branch points where $2 \operatorname{Tr}\left(\mathbf{M}^{2}\right)-\operatorname{Tr}(\mathbf{M})^{2}=8$.

(5) At $\mu=0$ the characteristic polynomial of the monodromy matrix has the following special form:

$$
\left.P(\lambda)\right|_{\mu=0}=1-\operatorname{Tr}(\mathbf{M}) \lambda+(2 \operatorname{Tr}(\mathbf{M})-2) \lambda^{2}-\operatorname{Tr}(\mathbf{M}) \lambda^{3}+\lambda^{4}
$$

Proof. That $\mu=0$ is always an eigenvalue follows from Noether's theorem and the phase invariance of NLS. The corresponding eigenvector is $p=\phi_{\text {stand }}(x), q=$ 0 . The fact that $\phi_{\text {stand }}$ is periodic (resp. antiperiodic) implies that $\mu=0$ is a periodic (resp. antiperiodic) eigenvalue. The fact that $\mathbf{M}$ is entire follows from standard arguments - see for example the text of Sibuya[25]. Note that $2 \operatorname{Tr}\left(\mathbf{M}^{2}\right)-$ 
$\operatorname{Tr}(\mathbf{M})^{2}=8$ is also an entire function of fractional order, and thus must have a countable number of zeros. The fact that $\mathbf{M}$ is symplectic for real $\mu$ follows from the results cited in the previous section. To see that this in fact holds for all $\mu \in \mathbf{C}$ simply note that $\mathbf{J}-\mathbf{M}^{t} \mathbf{J} \mathbf{M}$ is an entire function that is zero on the real axis, and thus must be identically zero[1]. From the first part it follows that $\lambda=1$ (resp. $\lambda=-1$ ) is a root of $\left.P(\lambda)\right|_{\mu=0}$. A simple division shows that the polynomial $\lambda-1$ divides the polynomial $\lambda^{4}+a \lambda^{3}+b \lambda^{2}+a \lambda+1$ if and only if $b+2 a+2=0$, which proves the last part. Note that this same calculation shows that if $\lambda=1$ is a root it is necessarily of multiplicity 2 or 4 . This is, again, due to the Hamiltonian symmetry.

LEMMA 3.2. If 0 is not a periodic eigenvalue of the $\mathbf{L}_{+}$operator the Floquet discriminants $K_{ \pm}(\mu)$ are analytic in a neighborhood of $\mu=0$.

Proof. For $\mu=0$ the stability problem decouples, and the monodromy matrix takes the block diagonal form

$$
\mathbf{M}=\left(\begin{array}{cc}
\mathbf{m}_{-} & 0 \\
0 & \mathbf{m}_{+}
\end{array}\right),
$$

where $\mathbf{m}_{ \pm}$are the monodromy matrices associated with $\mathbf{L}_{ \pm}$. It follows from the previous lemma that $\lambda=1$ is an eigenvalue of $\mathbf{m}_{-}$with multiplicity 2 . A short calculation using the fact that $2 \times 2$ matrices satisfy $\operatorname{Tr}\left(\mathbf{m}^{2}\right)=\operatorname{Tr}(\mathbf{m})^{2}-2 \operatorname{det}(\mathbf{m})$ shows that $2 \operatorname{Tr}\left(\mathbf{M}^{2}\right)-\operatorname{Tr}(\mathbf{M})^{2}+8=\left(\operatorname{Tr}\left(\mathbf{m}_{+}\right)-2\right)^{2}$. If 0 is not a periodic eigenvalue of $\mathbf{L}_{+}$then $\operatorname{Tr}\left(\mathbf{m}_{+}\right) \neq 2$, thus $2 \operatorname{Tr}\left(\mathbf{M}^{2}\right)-\operatorname{Tr}(\mathbf{M})^{2}+8 \neq 0$ and $K_{ \pm}$are analytic in a neighborhood of $\mu=0$.

THEOREM 3.3. A sufficient condition for the existence of a modulational instability spectrum is that $\mu=0$ is in the interior of a band of the $\mathbf{L}_{+}$operator.

Proof. From the previous lemma if $\mu=0$ is in the interior of a band of the $\mathbf{L}_{+}$ then the Floquet discriminants $K_{ \pm}(\mu)$ are analytic in a neighborhood of the origin. From the fact that the coefficients of the characteristic polynomial are invariant under the transformation $\mu \rightarrow-\mu$ it follows that $K_{ \pm}(\mu)$ are even, and thus are real on an segment of the imaginary axis containing the origin. Since $K_{-}(0) \in(-2,2)$ it follows that $K_{-}(\mu)$ is real and $\in(-2,2)$ on some segment of the imaginary axis containing the origin, and thus there is a modulational instability.

REMARK 3.4. The same argument guarantees the existence of a modulational instability if either $K_{+}(0)=+2$ and $K_{+}^{\prime \prime}(0) \geq 0$ or $K_{+}(0)=-2$ and $K_{+}^{\prime \prime}(0) \leq 0$. The calculation of the sign of the second derivative appears to require a difficult second order perturbation calculation.

3.2. Perturbative results for weak nonlinearity. In this section we study the Floquet spectrum for small amplitude waves, with a particular emphasis on the behavior near $\mu=0$. We shall see that the $\mathbb{U}(1)$ phase invariance of the NLS forces a four-fold degeneracy of the eigenvalues of the unperturbed monodromy matrix at $\mu=0$. Under perturbation this degeneracy can lead to the birth of a spine of continuous spectrum lying along the imaginary axis. Whether or not such a spine is born is determined by the relative sign of the nonlinearity and the quantity $\sigma$ defined in the previous section, and the length of the spine is of the order of $\sigma \epsilon$. 
When $\epsilon=0$ the operators $\mathbf{L}_{ \pm}$are equal and are given by

$$
\mathbf{L}_{-}(0)=\mathbf{L}_{+}(0)=-\frac{1}{2} \partial_{x x}+V(x)-\omega(0)
$$

and the resulting eigenvalue problem is self-adjoint. It is easy to see that in this case the spectrum of the stability problem is given by $\operatorname{spec}\left(\mathbf{L}_{-}\right) \cup \operatorname{spec}\left(-\mathbf{L}_{-}\right)$. In this case it is also straightforward to calculate that the monodromy matrix $\mathbf{M}$ of the full stability problem takes the block diagonal form

$$
\mathbf{M}=\left(\begin{array}{cc}
\mathbf{m}(\mu) & 0 \\
0 & \mathbf{m}(-\mu)
\end{array}\right)
$$

where $\mathbf{m}( \pm \mu)$ is the monodromy matrix for the problem

$$
\mathbf{L}_{-}(0) \psi= \pm \mu \psi \text {. }
$$

From this block diagonal form it follows that for $\epsilon=0$ the invariants of the full monodromy matrix can be expressed in terms of the Floquet discriminants $k( \pm \mu)$ of the second order problem via

$$
\begin{aligned}
\operatorname{Tr}(\mathbf{M}(\mu)) & =\operatorname{Tr}(\mathbf{m}(\mu))+\operatorname{Tr}(\mathbf{m}(-\mu))=k(\mu)+k(-\mu) \\
\operatorname{Tr}\left(\mathbf{M}^{2}(\mu)\right) & =\operatorname{Tr}\left(\mathbf{m}^{2}(\mu)\right)+\operatorname{Tr}\left(\mathbf{m}^{2}(-\mu)\right)=k^{2}(\mu)+k^{2}(-\mu)-4 .
\end{aligned}
$$

Here we have used the fact that $2 \times 2$ matrices satisfy the identity $\operatorname{Tr}\left(\mathbf{m}^{2}\right)=$ $\operatorname{Tr}(\mathbf{m})^{2}-2 \operatorname{det}(\mathbf{m})$. From this it follows that the Floquet discriminants $K_{ \pm}(\mu)$ for the full problem can be written in terms of the Floquet discriminant of $\mathbf{L}_{-}$via

$$
K_{ \pm}(\mu)=\frac{k(\mu)+k(-\mu) \pm \sqrt{(k(\mu)-k(-\mu))^{2}}}{2} .
$$

Obviously this could be simplified to $K_{ \pm}(\mu)=k( \pm \mu)$ however we do not do this, since it obscures the degeneracy at the points where $k(\mu)=k(-\mu)$.

The $\mathbb{U}(1)$ symmetry of the NLS equation implies that $\mu=0$ is a band-edge of $\mathbf{L}_{-}$. Thus for $\epsilon=0, \mu=0$ the monodromy matrix has has a single eigenvalue \pm 1 of multiplicity four. This corresponds to the last entry in the table in figure 1 . This seemingly non-generic four-fold degeneracy of eigenvalues is forced by the phaseinvariance symmetry along with the symplectic nature of the monodromy matrix, and can give rise to the modulational instability at non-zero wave amplitudes.

In the next lemma we present a normal form calculation for the Floquet discriminants in a neighborhood of $\mu=0, \epsilon=0$. The calculation is particularly simple due to some additional symmetries, which dramatically reduce the number of coefficients which need to be computed. We present the calculation for periodic band edges: the calculation for the anti-periodic bands edges is identical except for some sign changes. In this latter case we merely state the final result.

LEMMA 3.5. The Floquet discriminants of the monodromy matrix have the following normal form in a neighborhood of $\epsilon=0, \mu=0$ :

$$
\begin{gathered}
K_{ \pm}(\mu)=2+\frac{k^{\prime \prime}(0)}{2} \mu^{2}+2 \epsilon \sigma<\phi_{1}^{4}> \\
E_{1}=o\left(\epsilon, \mu_{1} \pm \sqrt{\left(k^{\prime}(0) \mu\right)^{2}+\left(2 \sigma \epsilon<\phi_{1}^{4}>\right)^{2}+E_{2}}\right. \\
E_{2}=o\left(\epsilon^{2}, \mu^{2}\right)
\end{gathered}
$$

where $k(\mu)$ is the Floquet discriminant for the $\mathbf{L}_{-}(0)$ operator. The band of unstable modes is, for small $\epsilon$, given by $\left(-i \frac{2 \sigma \epsilon<\phi_{1}^{4}>}{k^{\prime}(0)}, i \frac{2 \sigma \epsilon<\phi_{1}^{4}>}{k^{\prime}(0)}\right)$. 
Proof: This perturbation calculation is somewhat tedious but can be made simpler by the use of some of the previously derived identities. From the last part of Proposition 1 we can express the $\epsilon$ derivatives of the coefficients of the characteristic polynomial $P(\lambda)=\lambda^{4}+a(\mu, \epsilon) \lambda^{3}+b(\mu, \epsilon) \lambda^{2}+a(\mu, \epsilon) \lambda+1$ at the $\mu=0$ in terms of $\operatorname{Tr}(\mathbf{M} \mid \mu=0)$. This represents a considerable savings in effort, since we only need to calculate the four diagonal elements of $\mathbf{M}$, rather than all 16 elements of $\mathbf{M}$. To compute the $\mu$ derivatives of the coefficients of the characteristic polynomial at the origin we use the fact that $a(\mu, 0)=-(k(\mu)+k(-\mu)), b(\mu, 0)=k(\mu) k(-\mu)+2$. The mixed partial $\frac{\partial^{2}}{\partial \epsilon \partial \mu}$ vanishes at the origin since the $a, b$ coefficients are even functions of $\mu$ for all $\epsilon$. Note that, because of the square root branch point, it is necessary to carry the expansion out to second order to get what amounts to a first order result - the local normal form at the origin is a cone.

We present the calculation of $\frac{\partial \operatorname{Tr}(\mathbf{M} \mid \mu=0)}{\partial \epsilon}$ first. The problem $\mathbf{L}_{-}(\epsilon) \phi=0$ has a periodic solution that we denote by $\phi_{1}\left(=\phi_{\text {stand }}\right)$, and a linearly growing solution that we denote by $\phi_{2}$. We choose $\phi_{1,2}$ to form an orthogonal right-handed coordinate system:

$$
\begin{array}{rr}
\phi_{1}(0)=\cos (\theta) & \phi_{1}^{\prime}(0)=\sin (\theta) \\
\phi_{2}(0)=-\sin (\theta) & \phi_{2}^{\prime}(0)=\cos (\theta)
\end{array}
$$

Since $\mu=0$ is a band edge for the $\mathbf{L}_{-}(\epsilon)$ operator we have the following expressions for the period map:

$$
\begin{aligned}
\phi_{2}(1) & =\phi_{2}(0)+\sigma \phi_{1}(0)=\sigma \cos (\theta)-\sin (\theta) \\
\phi_{2}^{\prime}(1) & =\sigma \sin (\theta)+\cos (\theta),
\end{aligned}
$$

where $\sigma=\operatorname{Tr}(\mathbf{j m})$, with $\mathbf{m}$ the monodromy matrix for $\mathbf{L}_{-}(\epsilon)$ and $\mathbf{j}$ the Hamiltonian form. Again we emphasize that $\sigma$ must be non-zero if the band edge is not a double point, and the sign of $\sigma$ follows from the results of Lemma 2 .

It is convenient to define a second set of solutions $\psi_{1,2}$ which satisfy $\mathbf{L}_{-} \psi=0$ along with the initial conditions $\psi_{1}(0)=1, \psi_{1}^{\prime}(0)=0$ and $\psi_{2}(0)=0, \psi_{2}^{\prime}(0)=1$. These are obviously related to the $\phi_{1,2}$ by $\psi_{1}=\cos (\theta) \phi_{1}-\sin (\theta) \phi_{2}$ and $\psi_{2}=$ $\sin (\theta) \phi_{1}+\cos (\theta) \phi_{2}$. These functions form a natural basis in which to do perturbation theory on the $\mathbf{L}_{+}$operator. Note that we are considering $\mathbf{L}_{+}(\epsilon)$ as a perturbation of $\mathbf{L}_{-}(\epsilon)$, rather than $\mathbf{L}_{-}(0)$. This is slightly more convenient since, by phase invariance, we know exact eigenfunctions of the former.

As is usual in Floquet theory we must construct a fundamental set of solutions $\tilde{\psi}_{1}, \tilde{\psi}_{2}$ to

$$
\mathbf{L}_{+} \tilde{\psi}_{1,2}=\mathbf{L}_{-} \tilde{\psi}_{1,2}+2 \epsilon\left|\phi_{1}\right|^{2} \tilde{\psi}_{1,2}=0
$$

that satisfy the boundary conditions

$$
\begin{aligned}
& \tilde{\psi}_{1}(0)=1 \tilde{\psi}_{1}^{\prime}(0)=0 \\
& \tilde{\psi}_{2}(0)=0 \tilde{\psi}_{2}^{\prime}(0)=1
\end{aligned}
$$

A straightforward perturbation calculation gives the following expressions for the fundamental set of solutions to $\mathbf{L}_{+} \tilde{\phi}=0$ :

$$
\begin{aligned}
& \tilde{\psi}_{1}(x)=\psi_{1}-4 \epsilon\left(\phi_{1} \int \phi_{1}^{2} \phi_{2} \psi_{1}-\phi_{2} \int \phi_{1}^{3} \psi_{1}\right) \\
& \tilde{\psi}_{2}(x)=\psi_{2}-4 \epsilon\left(\phi_{1} \int \phi_{1}^{2} \phi_{2} \psi_{2}-\phi_{2} \int \phi_{1}^{3} \psi_{2}\right)
\end{aligned}
$$


Using the fact that $\phi_{2}(1)=\phi_{2}(0)+\sigma \phi_{1}(0)$ we get the following expression for the trace of the monodromy matrix to the leading order in $\epsilon$ in terms of $\phi_{1,2}$

$$
\begin{aligned}
\tilde{\psi}_{1}(1) & =\psi_{1}(1)-4 \epsilon\left(\cos ^{2}(\theta)\left(<\phi_{1}^{3} \phi_{2}>-\sigma<\phi_{1}^{4}>\right)\right. \\
& +\sin \theta \cos \theta\left(\sigma<\phi_{1}^{3} \phi_{2}>+<\phi_{1}^{4}>-<\phi_{1}^{2} \phi_{2}^{2}>\right) \\
& \left.-\sin ^{2}(\theta)<\phi_{1}^{3} \phi_{2}>\right) \\
\tilde{\psi}_{2}^{\prime}(1) & =\phi_{2}^{\prime}(1)-4 \epsilon\left(-\cos ^{2}(\theta)<\phi_{1}^{3} \phi_{2}>\right. \\
& \left.+\sin (\theta) \cos (\theta)<\phi_{1}^{2} \phi_{2}^{2}>-\sigma<\phi_{1}^{3} \phi_{2}>-<\phi_{1}^{4}>\right) \\
& +\sin ^{2} \theta\left(<\phi_{1}^{3} \phi_{2}>-\sigma<\phi_{1}^{4}>\right) .
\end{aligned}
$$

Here $<f>=\int_{0}^{1} f(y) d y$. From this it follows that the monodromy matrix is given to leading order in $\epsilon$ by

$$
\begin{aligned}
\operatorname{Tr}(\mathbf{M} \mid \mu=0) & =\tilde{\psi}_{1}(1)+\tilde{\psi}_{2}^{\prime}(1)+\psi_{1}(1)+\psi_{2}^{\prime}(1) \\
& =2\left(\psi_{1}(1)+\psi_{2}^{\prime}(1)\right)+4 \epsilon \sigma<\phi_{1}^{4}>+O\left(\epsilon^{2}\right) .
\end{aligned}
$$

It is easy to calculate that $a(\mu, 0)=-4-k^{\prime \prime}(0) \mu^{2}+o\left(\mu^{2}\right)$, and that $b(\mu, 0)=$ $6+\left(2 k^{\prime \prime}(0)-\left(k^{\prime}(0)\right)^{2}\right) \mu^{2}+o\left(\mu^{2}\right)$. This, together with the vanishing of the mixed partial and the equation (2.11) gives the above result.

REMARK 3.6. From the proof of the preceeding lemma, and the use of the time-invariant Hamiltonian energy functional

$$
\mathbb{H}=\int\left(\frac{1}{2}\left|\psi_{x}\right|^{2} \pm \frac{\epsilon}{2}|\psi|^{4}-V(x)|\psi|^{2}\right) d x,
$$

it follows that

$$
\left.\frac{\partial \operatorname{Tr} \mathbf{M}}{\partial \epsilon}\right|_{(0,0)}=\left.8 \sigma \frac{\partial \mathbb{H}}{\partial \epsilon}\right|_{\epsilon=0} .
$$

Thus, the instability condition can be expressed in terms of the signs of $\sigma$ and of the derivative of the energy functional. This is a feature that appears regularly in the study of stability of nonlinear waves: that the sign of the derivative of some conserved quantity is a proxy for the index of some linearized operator. For some results of a similar flavor in a variety of different contexts (both conservative and dissipative) see $[\mathbf{9}, \mathbf{1 1}, \mathbf{1 2}, \mathbf{1 8}, \mathbf{1 9}]$.

THEOREM 3.7. For $\epsilon$ small and positive (focusing NLS) and solutions bifurcating from a lower band edge that is not a double point, or for $\epsilon$ small and negative and solutions bifurcating from an upper band edge that is not a double point there exists a band of spectrum along the imaginary axis.

Proof. The proof follows from the preceeding lemma and the the first theorem. From the explicit factorization of the characteristic polynomial it follows that, for $\epsilon$ fixed, the Floquet discriminants $K_{ \pm}(\mu)$ are analytic functions in the cut plane with branch points at the points where

$$
2 \operatorname{Tr}\left(\mathbf{M}^{2}\right)-\operatorname{Tr}(\mathbf{M})^{2}+8=0
$$

From the preceeding lemma it follows that at $\mu=0$ this quantity reduces to

$$
\left.\left(2 \operatorname{Tr}\left(\mathbf{M}^{2}\right)-\operatorname{Tr}(\mathbf{M})^{2}+8\right)\right|_{\mu=0}=\left(\left.\operatorname{Tr}(\mathbf{M})\right|_{\mu=0}-4\right)^{2}=16 \sigma^{2} \epsilon^{2}<\psi_{1}^{4}>^{2}+o\left(\epsilon^{2}\right)
$$


and thus this quantity is nonzero for small but nonzero $\epsilon$ : the fact that the band edge is not a double point guarantees that $\sigma \neq 0$. It follows that the Floquet discriminants $K_{ \pm}$are analytic in a neighborhood of $\mu=0$ and satisfy

$$
\begin{aligned}
& K_{+}(0)=2+4 \epsilon \sigma<\psi_{1}^{4}>+O\left(\epsilon^{2}\right) \\
& K_{-}(0)=2 .
\end{aligned}
$$

As in the first theorem the fact that $K_{+}(\mu)$ is an even function of $\mu$ implies that $\mu=$ 0 is a critical point, and $K_{+}$is real on a segment of the imaginary axis containing the origin. Recall that for odd numbered bands the periodic eigenvalue is the lower band edge, and $\sigma$ is positive, and for even numbered bands the periodic eigenvalue is the upper band edge, and $\sigma$ is negative. For $\epsilon<0$ (defocusing) and odd numbered bands, or for $\epsilon>0$ and even numbered bands the perturbation result implies that $K_{+}(0) \in(-2,2)$, so there exists an interval on the imaginary axis on which $K_{+}(\mu)$ is real and $\in(-2,2)$. The analogous calculation for solutions bifurcating from the anti-periodic eigenvalues shows that for $\epsilon<0$ (defocusing) and even numbered bands, for $\epsilon>0$ and odd numbered bands $K_{+}(0) \in(-2,2)$, and there exists a spine of spectrum along the imaginary axis. Thus, for $\epsilon$ small and defocusing nonlinearity standing wave solutions which bifurcate from the lower band edge are modulationally unstable, while for focusing nonlinearity standing wave solutions which bifurcate from the upper band edge are modulationally unstable.

Note that if $K_{ \pm}^{\prime \prime}(0)=0$ there exist additional arcs of spectrum emerging from the origin into the complex plane. For $\epsilon$ sufficiently small the local normal form calculation guarantees that the second derivative is non-vanishing, and this does not occur. It remains a possibility for larger $\epsilon$, though we have not been able to observe it in numerical experiments.

\section{Finite Wavelength Instabilities.}

The instability considered in the previous section is a long-wavelength, or modulational, instability. The wavelength of the unperturbed solution is marginally stable, but a band of wavelengths around this wavelength are unstable, and the spectrum consists of a band of unstable eigenvalues containing the origin. In this section we consider the possibility of finite wavelength instabilities, where the band of unstable eigenvalues does not contain the origin. This case is somewhat more difficult than the case of modulational instabilities, since there are fewer symmetries, and our results are somewhat less detailed. In addition to the analyticity arguments of the previous section a major tool will be the Krein signature.

First we note the following lemma

LEMMA 4.1. The spectrum of the stability problem consists of a union of continuous curves in the complex plane. The possible endpoints of the curves are periodic/antiperiodic points $K_{ \pm}(\mu)= \pm 2$, critical points $K_{ \pm}^{\prime}(\mu)=0$, or branch points $a^{2}(\mu)-4 b(\mu)+8=0$ (or $\left.K_{+}(\mu)=K_{-}(\mu)\right)$.

ProOF. As before $K_{ \pm}(\mu)$ are analytic away from the (isolated) points where $a^{2}(\mu)-4 b(\mu)+8=0$. Suppose $\mu$ is a point in the spectrum that is not a branch point, (anti)-periodic point or critical point. We assume for the sake of argument that $K_{+}(\mu) \in(-2,2)$. Since $K_{+}$is analytic in a neighborhood of $\mu$ and $\mu$ is not a critical point of $K_{+}$an appeal to the implicit function theorem shows the existence 
of a unique curve through $\mu$ along which $K_{+}$is real. Since $\mu$ is not a band edge continuity implies that $K_{+}$is real and $\in(-2,2)$ in some neighborhood of $\mu$.

Remark: Note that for Hill's equation the only possible band edges are periodic or anti-periodic points - the first possibility. For non-self-adjoint Schrodinger operators ( as noted by Shin[24]) the second possibility arises. The third possibility only arises for equations of higher order than second.

We now consider the possibility of finite wavelength instabilities arising from points $\mu^{*}$ where $K_{+}\left(\mu^{*}\right)=K_{-}\left(\mu^{*}\right)$. First note that in the small amplitude limit, when $\epsilon=0$, we have $a^{2}-4 b+8=(k(\mu)-k(-\mu))^{2}=k^{\prime \prime}\left(\mu^{*}\right)\left(\mu-\mu^{*}\right)^{2}+O\left(\left(\mu-\mu^{*}\right)^{4}\right)$ so the branch points are degenerate (once again the normal form is a cone). Under perturbation this degeneracy is expected to break, and generically it can do so in one of two ways, which are generally referred to as the avoided collision and the open gap. These possibilities are illustrated in figure 1, along with a picture of the spectrum in the complex plane. Note that the curves of spectrum in the imaginary direction emerge from the critical points $K_{ \pm}^{\prime}(\mu)=0$, while the edges of the gap occur at the branch points, where $K_{ \pm}^{\prime}(\mu)=\infty$, so the real spectrum protrudes slightly into the interior of the eye.

It is rather tedious to compute the normal form for this case, since one lacks the even symmetry, but consideration of the Krein-Gelfand-Lidskii theorem can reduce the possibilities. In the case of a collision of two eigenvalues with the same Krein sign the open gap is forbidden, since that corresponds to eigenvalues leaving the unit circle. In the case of two eigenvalues with the opposite Krein sign one expects that under perturbation the eigenvalues should leave the unit circle. We formalize this observation as a theorem.

THEOREM 4.2. In the neighborhood of an accidental degeneracy $\left(\mu=\mu^{*}, \epsilon=0\right)$ the Floquet discriminants have the following normal form

$$
\begin{gathered}
K_{ \pm}(\mu)=k\left(\mu^{*}\right)+\left(k^{\prime}\left(\mu^{*}\right)-k^{\prime}\left(-\mu^{*}\right)\right)\left(\mu-\mu^{*}\right)+\alpha \epsilon+E_{1} \pm \\
\sqrt{\left(k^{\prime}\left(\mu^{*}\right)+k^{\prime}\left(-\mu^{*}\right)\right)^{2}\left(\mu-\mu^{*}\right)^{2}+\gamma \epsilon^{2}+E_{2}} \\
E_{1}=O\left(\epsilon^{2},\left(\mu-\mu^{*}\right)^{2}, \epsilon\left(\mu-\mu^{*}\right)\right) \\
E_{1}=O\left(\epsilon^{3},\left(\mu-\mu^{*}\right)^{3}, \epsilon\left(\mu-\mu^{*}\right)^{2}, \epsilon^{2}\left(\mu-\mu^{*}\right)\right)
\end{gathered}
$$

Further, if $\operatorname{sgn}\left(k^{\prime}\left(\mu^{*}\right)\right)=\operatorname{sgn}\left(k^{\prime}\left(-\mu^{*}\right)\right)$ the quadratic form $\left(k^{\prime}\left(\mu^{*}\right)+k^{\prime}\left(-\mu^{*}\right)\right)^{2}(\mu-$ $\left.\mu^{*}\right)^{2}+\gamma \epsilon^{2}$ is nonnegative.

Proof. A straightforward, though slightly tedious, perturbation argument shows that $\left.\frac{\partial}{\partial \epsilon}\left(a^{2}-4 b+8\right)\right|_{\epsilon=0}=0$, so that this quantity is locally quadratic, as well as having a vanishing mixed partial at $\epsilon=0$. The Krein signs of the unperturbed eigenvalues are the same as the signs of $k^{\prime}(\mu)$ and $k^{\prime}(-\mu)$ respectively. In the case where these signs are the same the Krein-Gelfand-Lidskii theorem guarantees that, under small perturbations, the eigenvalues of $\mathbf{M}$ remain on the unit circle. Since non-negativity of the above quadratic form is a necessary condition for the eigenvalues to remain on the unit circle the the sign condition together with the Krein theorem guarantees non-negativity of this form.

Note that this theorem does not preclude the following possibilities: in the case of like Krein sign collisions it is possible that the quadratic form is only semidefinite, rather than being strictly positive. Such would be the case if the intersection persists under perturbation. In the case of collisions of opposite Krein sign a 
naive application of the Krein-Gelfand-Lidskii theorem gives no information. While the Krein-Gelfand-Lidskii theorem implies that generic perturbations should cause eigenvalues of opposite Krein sign to move off of the unit circle there is no guarantee that this particular perturbation will do so. We conjecture that, in the small amplitude case the signature of the quadratic form is equal to the signature of the subspace. This would imply that that intersections of opposite Krein signs always open to a gap, while intersections of the same Krein sign always open to an avoided collision. Very preliminary numerical evidence has supports this conjecture, but we currently have not proof of this. FInally we note that $\mu=0$ always corresponds to a collision of eigenvalues of like Krein sign, implying that this degeneracy will generically open to the avoided collision, in agreement with the results of Lemma 5. In Fig(1) we illustrate these possibilities: an intersection of Floquet discriminants of the opposite slope opening to an avoided collision, and an intersection of discriminants of like slope opening to a gap. We have not illustrated the intersection of Floquet discriminants of the opposite slope opening to a gap, which is forbidden by the Krein theorem, or the intersection of Floquet discriminants of the same slope opening to an avoided collision, which is non-generic if it occurs at all.

An obvious corollary of this is the following:

Corollary 4.3. If $\operatorname{sgn}\left(k^{\prime}\left(\mu^{*}\right)\right)=\operatorname{sgn}\left(k^{\prime}\left(-\mu^{*}\right)\right)$ and $\gamma>0$, or if $\operatorname{sgn}\left(k^{\prime}\left(\mu^{*}\right)\right)=$ $-\operatorname{sgn}\left(k^{\prime}\left(-\mu^{*}\right)\right)$ and $\gamma<0$ then there exists a band of spectrum off of the real axis.

From the local normal form it is an easy calculation that, in either case, for sufficiently small $\epsilon$ the Floquet discriminants $K_{ \pm}$must have a critical point in the neighborhood of $\mu^{*}$, and that $a^{2}-4 b+8 \neq 0$ at this critical point. From the analyticity arguments of the previous section this guarantees that $K_{ \pm}$are real and $\in(-2,2)$ in some neighborhood of the critical point. Again one expects that in the case of a like Krein sign collision one should generically have $\gamma>0$, leading to the avoided collision and a loop of spectrum opening into the complex plane, but there seems to way to show this in any particular case without actually calculating $\gamma$. It is interesting that the cases which would not lead to the spectrum opening into the complex plane are the ones that are disallowed or non-generic.

\section{Explicit Examples and Numerics}

In this section we present some examples. We will primarily be working with known exact elliptic function solutions. We consider the nonlinear Schrödinger equation

$$
i \psi_{t}=-\frac{1}{2} \psi_{x x}+V_{0} \operatorname{sn}^{2}(x, k) \psi \pm|\psi|^{2} \psi
$$

This equation has a one-parameter family of exact solutions given by

$$
\begin{aligned}
\psi(x, t) & =r(x) e^{-i \omega t+i \theta(x)} \\
r^{2}(x) & =A \operatorname{sn}^{2}(x, k)+B \\
\theta(x) & =c \int \frac{d x^{\prime}}{r^{2}\left(x^{\prime}\right)} \\
A & =-\left(V_{0}+k^{2}\right) \\
c^{2} & =B\left(\frac{B}{V_{0}+k^{2}-1}\right)\left(V_{0}+k^{2}-B k^{2}\right)
\end{aligned}
$$


Where $B \in\left(-\infty,-k^{2}\right) \cup\left(\frac{V_{0}+k^{2}}{k^{2}}, V_{0}+k^{2}\right)$ for the focusing sign and $B \in\left(-\left(V_{0}+k^{2}\right),-\frac{V_{0}+k^{2}}{k^{2}}\right) \cup$ $\left(-k^{2}, \infty\right)$ for the defocusing sign. These solutions represent nonlinear stationary states which bifurcate from the linear Bloch states. We are primarily interested in the solutions which bifurcate from the band edges. These correspond to the boundaries of the above regions of validity. In the focusing case we have

$$
\begin{aligned}
& r_{0}(x)=\frac{\sqrt{V_{0}+k^{2}}}{k^{2}} \operatorname{dn}(x, k) \omega=-1-\frac{V_{0}}{k^{2}}+\frac{k^{2}}{2} V_{0}+k^{2}>0 \\
& r_{1}(x)=\sqrt{V_{0}+k^{2}} \operatorname{cn}(x, k) \omega=\frac{1}{2}-V_{0}-k^{2} v_{0}+k^{2}>0 \\
& r_{2}(x)=\sqrt{-\left(V_{0}+k^{2}\right)} \operatorname{sn}(x, k) \omega=\frac{1+k^{2}}{2} V_{0}+k^{2}<0,
\end{aligned}
$$

while in the defocusing case we have the analogous solutions

$$
\begin{aligned}
& r_{0}(x)=\frac{\sqrt{V_{0}+k^{2}}}{k^{2}} \operatorname{dn}(x, k) \omega=-1-\frac{V_{0}}{k^{2}}+\frac{k^{2}}{2} V_{0}+k^{2}<0 \\
& r_{1}(x)=\sqrt{V_{0}+k^{2}} \operatorname{cn}(x, k) \omega=\frac{1}{2}-V_{0}-k^{2} v_{0}+k^{2}<0 \\
& r_{2}(x)=\sqrt{-\left(V_{0}+k^{2}\right)} \operatorname{sn}(x, k) \omega=\frac{1+k^{2}}{2} V_{0}+k^{2}>0 .
\end{aligned}
$$

The solutions all bifurcate from the the linear Bloch states at $V_{0}+k^{2}=0$, with the $\mathrm{dn}, \mathrm{cn}$ solutions existing on one side of the bifurcation and the sn solution on the other side. It is easy to check that the spectrum of the $\mathbf{L}_{-}$operator in each of these cases is the 1-gap Lamé operator plus some constant which differs in each case. The ground state of $\mathbf{L}_{-}$is $\operatorname{dn}(x, k)$, while the next two antiperiodic eigenfunctions are given by $\operatorname{cn}(x, k), \operatorname{sn}(x, k)$. The gap between the eigenvalues corresponding to the $\mathrm{cn}$ and sn is the only open gap - the remainder of the gaps are closed to double points. The values of $\sigma$ correspoding to each of these band edges can be computed explicitly. They are given explicitly in terms of elliptic integrals as follows:

$$
\begin{aligned}
\sigma_{\mathrm{dn}} & =\int_{0}^{2 K} \frac{d x}{\mathrm{dn}^{2}(x, k)}=2 \int_{\sqrt{1-k^{2}}}^{1} \frac{d y}{y^{2} \sqrt{\left(1-y^{2}\right)\left(y^{2}-\left(1-k^{2}\right)\right)}} \\
\sigma_{\mathrm{cn}} & =k^{2} \int_{0}^{2 K} \frac{\operatorname{sn}^{2}(x, k)}{\operatorname{dn}^{2}(x, k)} d x=2 \int_{\sqrt{1-k^{2}}}^{1} \frac{\left(1-y^{2}\right)}{y^{2} \sqrt{\left(1-y^{2}\right)\left(y^{2}-\left(1-k^{2}\right)\right)}} d y \\
\sigma_{\mathrm{sn}} & =-k^{2} \int_{0}^{2 K} \operatorname{sn}^{2}(x, k) d x=-2 \int_{\sqrt{1-k^{2}}}^{1} \frac{\left(1-y^{2}\right)}{\sqrt{\left(1-y^{2}\right)\left(y^{2}-\left(1-k^{2}\right)\right)}} d y .
\end{aligned}
$$

The $\sigma_{\mathrm{dn}}$ can be computed using the usual reduction of order technique to compute the second linearly independent solution. A similar calculation gives $\sigma_{\mathrm{cn}, \mathrm{sn}}$, but one must deform the integral into the complex plane to avoid poles of the elliptic functions. Note that $\sigma_{\mathrm{dn}}$ and $\sigma_{\mathrm{cn}}$ have the same sign, as they are band edges of the first band, while $\sigma_{\mathrm{sn}}$ has the opposite sign, since it is the band edge of the second band.

In what follows the parameter $\epsilon=V_{0}+k^{2}$. There is an obvious rescaling which transforms the above form of the NLS equation to the form considered earlier.

5.1. Jacobi sine $(\operatorname{sn}(x, k))$ type solutions. It follows from Theorem $(2)$ that for $V_{0}+k^{2}=\epsilon$ suffiently small that the dn and sn solutions, as lower band edges, are modulationally unstable. Moreover, from Theorem (1) we are guaranteed a 

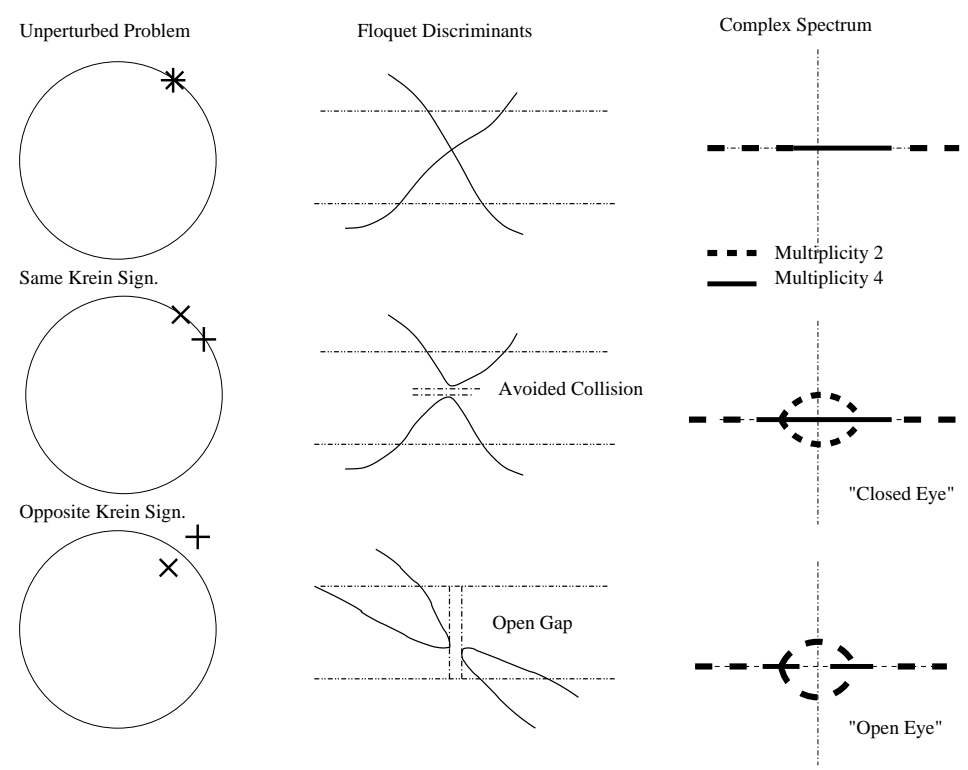

FiguRE 1. The possible bifurcations of degenerate eigenvalues of a symplectic matrix under perturbation, and the local behavior of the Floquet discriminants.

modulational instability as long as the $\mathbf{L}_{+}$operator is in the interior of a band at $\mu=0$. Thus in each case we have an open interval $\left(0, \epsilon^{*}\right)$ in which we are guaranteed a modulational instability, where $\epsilon^{*}$ is the smallest positive value of $\epsilon$ such that $\mu=0$ is a band edge of the $\mathbf{L}_{+}$operator. We will focus on the sn solution, since the instability of the dn solution follows from more elementary arguments. We have found numerically that $\mu=0$ is in the interior of a band for $\epsilon \in(-1.33,0) \cup(-3.0,-1.56) \cup(-6.7,-5.6) \ldots$, which shows instability for $\epsilon$ in these intervals. The numerical evidence further shows that in this particular case the second derivative of the branch of the Floquet discriminant passing through -2 is always negative, which implies that these solutions are always unstable. We do not currently have a proof of this. It is also whether this feature is special to the elliptic function solutions, or if it holds in greater generality. It is interesting to note that the $\operatorname{sn}(x, k)$ solution also has a finite-wavelength instability that appears at arbitrarily small positive amplitude. This is illustrated in Fig (2). When $\epsilon=0$ the Floquet discriminants of the unperturbed problem $k( \pm \mu)$ intersect near $\mu \approx .45$. This is a collision of opposite Krein sign, since the discriminants have slopes of the same sign. This intersection opens into a gap under perturbation, as is clear from the figure. In Fig(3) we show a detail of the previous figure, along with a grey-scale plot of the size of the imaginary part of the Floquet discriminant, with black representing a negative imaginary part and white a positive imaginary part. The curve of real $K_{+}(\mu)$ ) is clearly visible as a sharp edge. Careful inspection of this graph shows that these additional branches of spectrum emerge from the real axis at the critical points $K_{ \pm}^{\prime}(\mu)=0$. 

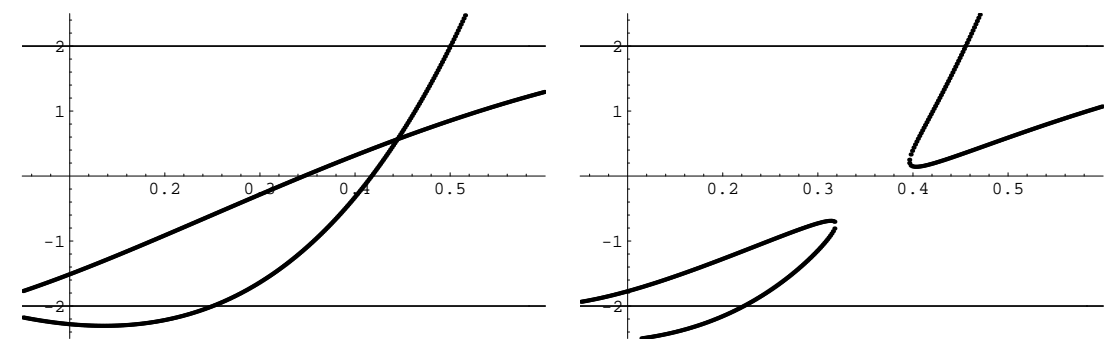

Figure 2. The Floquet Discriminants for the $\operatorname{sn}(x, k)$ solution in the focusing case with $\epsilon=0, .1$
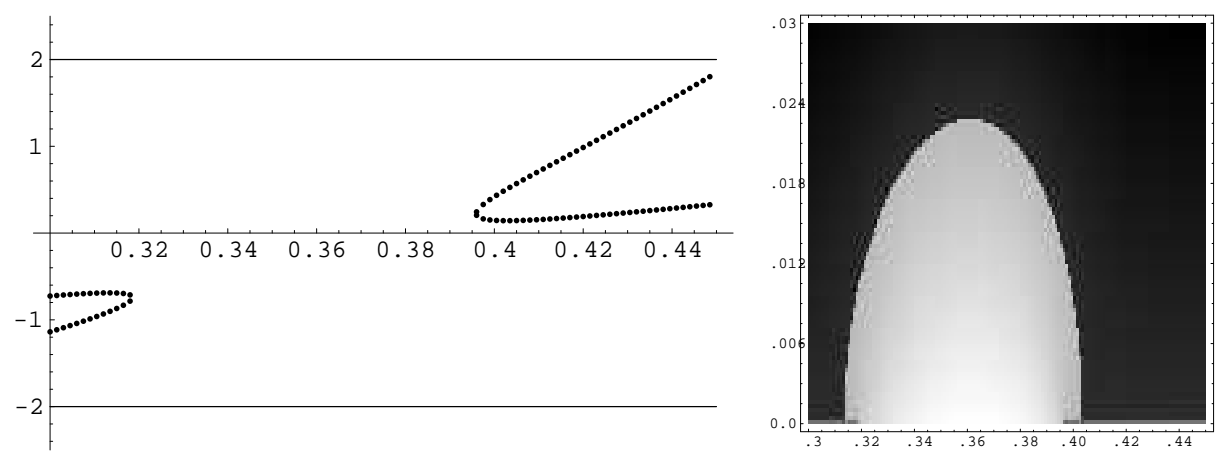

Figure 3. A detail of the previous figure. The first graph shows the Floquet discriminants along the real $\mu$ axis for $\epsilon=.1$ (note the open gap) and the second graph shows a greyscale plot of the imaginary part of the Floquet discriminant in the complex plane.
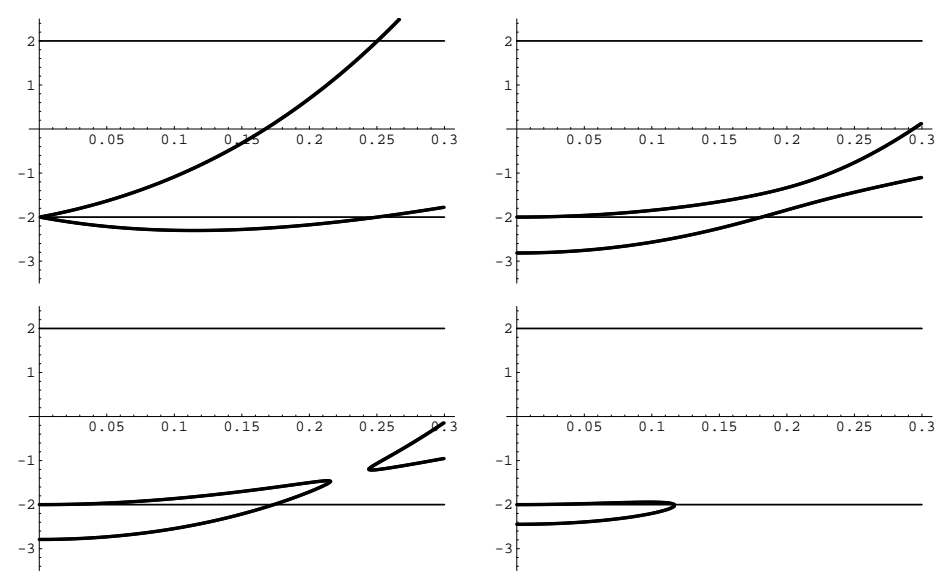

Figure 4. The Floquet discriminants for the focusing $\operatorname{cn}(x, k)$ solution for $V_{0}=-1 / 2,-1 / 4,-.228,-.1$. 
5.2. Jacobi cosine $(\mathrm{cn}(x, k))$ type solutions: The stability of the cn type solutions is extremely interesting, and is the subject of Fig(4). For small $\epsilon$ the perturbation result guarantees that $\mu=0$ is in a gap for the $\mathbf{L}_{+}$operator, and numerically we find no modulational instability for small $\epsilon$. As before we expect that for some critical value of $\epsilon \mu=0$ will enter a band of $\mathbf{L}_{+}$and an instability will develop. In this case the critical value of $\epsilon$ can actually be computed explicitly. When $V_{0}=0\left(\epsilon=k^{2}\right)$ we find that $\mathbf{L}_{+}$is the 2-gap Lamé operator, with $\mu=0$ is a band edge. It is also straightforward to show using the Sturm oscillation theorem and monotonicity of the band edges in $\epsilon$ that this is the smallest value of $\epsilon$ for which $\mathbf{L}_{+}$has a band edge at $\mu=0$. Thus we are guaranteed a modulational instability for some interval of $\epsilon$ beginning at $V_{0}=0\left(\epsilon=k^{2}\right)$. It is worth remarking that in this critical case (the integrable one) all of the information about the spectrum of the linearization, as well as a great deal more, can be obtained as degenerate cases of exact N-gap solutions to NLS. See, in particular, Chapter 4 of the text by Belokolos, Bobenko, Enol'skii, Its and Matveev[2] for the general case, or the work of Ercolani, Forest and McLaughlin[6, 7] for the degenerate 1-gap case.

The birth of a modulational instability at $V_{0}=0$ supports a intuition based on physical reasoning that was put forth in $[3]$ which suggested that such solutions should go unstable at $V_{0}=0$. Interestingly it appears that there exists a finite amplitude side-band type instability that sets in before the modulational instability. This is illustrated in figure 4, which shows the Floquet discriminants for the linearized operator of the focusing NLS about a cn type solution with modulus $k^{2}=1 / 2$ and a sequence of different values of $\epsilon$. Near $V_{0} \approx-.23$ the Floquet discriminants $K_{ \pm}$cross, causing a gap to open and a loop of spectrum to emerge into the complex plane. This instability disproves a guess made in [3], based on numerical experiments, that the cn type solutions should be stable for $V_{0}<0$. As $V_{0}$ increases the neighborhood about the origin in which the Floquet discriminants are real shrinks, until at $V_{0}=0$ the discriminants are only real at the origin. For positive $V_{0}$ the discriminants again become real in a neighborhood of the origin, and the results of previous sections imply the existence of a modulational instability.

\section{Conclusions}

We have established a sufficient condition for the modulational instability of a periodic standing wave solution to the NLS equation, which can be easily checked. In the case of weak nonlinearity this reduces to a physically reasonable criterion based on the effective mass of a particle in the periodic potential. We have also made some preliminary progress into studying the birth of side-band type instabilities. In the case of like Krein-sign collisions the gap-opening bifurcation is forbidden. Very preliminary numerical experiments suggest that, in the case of opposite Krein-sign collisions the "avoided collision" bifurcation is forbidden, but we do not currently have a proof of this.

It is interesting to compare these results to the analogous theory for decaying potentials on the whole line. In previous work Rose and Weinstein[22] gave a detailed analysis of the problem

$$
i \psi_{t}=-\frac{1}{2} \psi_{x x}+V(x) \psi-|\psi|^{2} \psi
$$

under the assumption that $V(x)$ is decaying at infinity. They were able to show the existence of a family of stationary solutions, which arise as minimizers of a 
certain functional. These solutions bifurcate from the ground state of the linear problem. Further, they showed that these solutions are stable for sufficiently small amplitudes. In contrast our results show that in the periodic case the focusing NLS solutions which bifurcate from the ground state (necessarily a lower band edge) are always unstable for sufficiently small amplitudes. This is presumably a reflection of the fact that in the whole line case there is always a gap between the ground state energy and the energy of the next excited state, while in the periodic case one has a continuum of energies, but it would be nice to have a more detailed understanding of this point.

\section{References}

[1] Lars V. Ahlfors. Complex analysis. McGraw-Hill Book Co., New York, third edition, 1978. An introduction to the theory of analytic functions of one complex variable, International Series in Pure and Applied Mathematics.

[2] E.D. Beolokolos, A.I. Bobenko, V.Z. Enol'skii, A.R. Its, and V.B Matveev. Algebro-geometric approach to nonlinear integrable equations. Springer-Verlag, Berlin, 1994.

[3] J.C. Bronski, L.D. Carr, R. Carretero-Gonzalez, B. Deconinck, J.N. Kutz, and K. Promislow. Stability of attractive Bose-Einstein condensates in a periodic potential. Phys. Rev E., 64:056615, 2001.

[4] J.D. Carter and H. Segur. Instabilities in the two-dimensional cubic nls equation. Phys. Rev. E, 68:045601, 2003.

[5] Michael G. Crandall and Paul H. Rabinowitz. Nonlinear Sturm-Liouville eigenvalue problems and topological degree. J. Math. Mech., 19:1083-1102, 1969/1970.

[6] N. Ercolani, M. G. Forest, and D. W. McLaughlin. The origin and saturation of modulational instabilities. Phys. D, 18(1-3):472-474, 1986. Solitons and coherent structures (Santa Barbara, Calif., 1985).

[7] N. Ercolani, M. G. Forest, and David W. McLaughlin. Geometry of the modulational instability. III. Homoclinic orbits for the periodic sine-Gordon equation. Phys. D, 43(2-3):349-384, 1990.

[8] M Grillakis. Analysis of the linearization around a critical point of an infinite-dimensional hamiltonian system. Comm. Pure Appl. Math., 1990.

[9] M. Grillakis, J. Shatah, and W. Strauss. Stability theory of solitary waves in the presence of symmetry. I,II. J. Funct. Anal., 74(1):160-197,308-348, 1987.

[10] C.K.R.T Jones. Instability of standing waves for nonlinear schr?dinger-type equations. Ergodic Theory Dynam. Systems, 1988.

[11] Todd Kapitula. Stability criterion for bright solitary waves of the perturbed cubic-quintic Schrödinger equation. Phys. D, 116(1-2):95-120, 1998.

[12] R. S. Laugesen and M. C. Pugh. Linear stability of steady states for thin film and CahnHilliard type equations. Arch. Ration. Mech. Anal., 154(1):3-51, 2000.

[13] Y. Li and David W. McLaughlin. Morse and Melnikov functions for NLS PDEs. Comm. Math. Phys., 162(1):175-214, 1994.

[14] R. S. MacKay and P. G. Saffman. Stability of water waves. Proc. Roy. Soc. London Ser. A, 406(1830):115-125, 1986.

[15] R.S. MacKay. Stability of equilibria of hamiltonian systems. In Sarben Sarkar, editor, Proceedings of the seminar on dynamical systems held in Malvern, April 23-25, 1985, Malvern Physics Series, pages 254-270, Bristol, 1986. Adam Hilger Ltd.

[16] Wilhelm Magnus and Stanley Winkler. Hill's equation. Dover Publications Inc., New York, 1979. Corrected reprint of the 1966 edition.

[17] Boris Mityagin. Spectral expansions of one-dimensional periodic Dirac operators. Dyn. Partial Differ. Equ., 1(2):125-191, 2004.

[18] M. Oh and K. Zumbrun. Stability of periodic solutions of conservation laws with viscosity: pointwise bounds on the Green function. Arch. Ration. Mech. Anal., 166(2):167-196, 2003.

[19] Robert L. Pego and Michael I. Weinstein. Eigenvalues, and instabilities of solitary waves. Philos. Trans. Roy. Soc. London Ser. A, 340(1656):47-94, 1992.

[20] D.E. Pelinovsky, A.A. Sukhorukov, and Yu. S. Kivshar. Bifurcations and stability of gap solitons in periodic potentials. Phys. Rev. E, 2004. 
[21] Paul H. Rabinowitz. Nonlinear Sturm-Liouville problems for second order ordinary differential equations. Comm. Pure Appl. Math., 23:939-961, 1970.

[22] H. A. Rose and M. I. Weinstein. On the bound states of the nonlinear Schrödinger equation with a linear potential. Phys. D, 30(1-2):207-218, 1988.

[23] B. Sandstede and A. Yew. Personal Communications.

[24] Kwang C. Shin. On the shape of spectra for non-self-adjoint periodic Schrödinger operators. J. Phys. A, 37(34):8287-8291, 2004.

[25] Yasutaka Sibuya. Linear differential equations in the complex domain: problems of analytic continuation, volume 82 of Translations of Mathematical Monographs. American Mathematical Society, Providence, RI, 1990. Translated from the Japanese by the author.

[26] B. Wu and Q. Niu. Superfluidity of bose-einstein condensate in an optical lattice: Landauzener tunnelling and dynamical instability. New J. Physics, 104(5), 2003.

[27] V. A. Yakubovich and V. M. Starzhinskii. Linear differential equations with periodic coefficients. 1, 2. Halsted Press [John Wiley \& Sons] New York-Toronto, Ont.,, 1975. Translated from Russian by D. Louvish.

Department of Mathematics, University of Illinois Urbana-Champaign, Urbana, IL 61801

E-mail address: jared@math.uiuc.edu

School of Mathematics, Institute for Advanced Study, Princeton, NJ 08540

Current address: Department of Mathematics, University of Illinois Urbana-Champaign, Urbana, IL 61801

E-mail address: zrapti@math.uiuc.edu 